\title{
AUTOMATIC PARAMETERIZATION OF GREY-LEVEL HIT-OR-MISS OPERATORS FOR BRAIN VESSEL SEGMENTATION
}

\author{
N. Passat, C. Ronse \\ LSIIT, UMR 7005 CNRS-ULP \\ Strasbourg I University \\ Illkirch-Graffenstaden, France
}

\author{
J. Baruthio, J.-P. Armspach \\ IPB, UMR 7004 CNRS-ULP \\ Strasbourg I University \\ Strasbourg, France
}

\begin{abstract}
Reliable segmentation of 3D magnetic resonance angiography (MRA) is fundamental for planning and performing neurosurgical procedures, but also for detecting vascular pathologies. We propose here a method for brain vessel segmentation based on mathematical morphology tools. This method, devoted to phase-contrast MRA (PC-MRA) performs vessel segmentation by applying an adaptive set of grey-level hit-or-miss operators on each point of the MR data. High level anatomical knowledge modeled by a vascular atlas is used in order to adapt the parameters of these operators (number, size, and orientation) to the current position. The method has been performed on 30 PC-MRA cases composed of both phase and magnitude images. The results have been validated and compared to segmented data obtained by applying a region-growing algorithm on the same database. They tend to prove that the method is reliable for brain vessel detection and additionnally provides information on vessel size and orientation without requiring any post-processing step.
\end{abstract}

\section{INTRODUCTION}

Phase-contrast magnetic resonance angiography (PC-MRA) is a technique frequently used to provide $3 \mathrm{D}$ images of cerebral vascular structures. Indeed, the availability of precise information about brain vascular networks is fundamental for planning and performing neurosurgical procedures, but also for detecting aneurysms and stenoses. Many classical image processing tools have already been applied to the case of vessel segmentation. Nevertheless, nearly all the proposed algorithms use very little a priori knowledge and do not process multimodal data. In a previous paper [1] we proposed a first attempt to use anatomical knowledge to perform a segmentation on PC-MRA bimodal data. In this paper, we propose a new algorithm based on grey-level hit-ormiss transform using structuring elements designed accord-

Thanks to the Équipe-Projet Multi-Laboratoires Imagerie et Robotique Médicale et Chirurgicale (EPML \#9 CNRS-STIC) for funding. ing to high level anatomical knowledge. This knowledge has been modeled by creating a vascular atlas from a set of segmented PC-MRA. This paper is organized as follows. Section 2 reviews some of the previous vessel segmentation techniques. Section 3 provides details on PC-MRA acquisitions. Sections 4 and 5 describe the anatomical and theoretical background necessary to understand and justify the algorithm proposed in Section 6. In Section 7, the method is tested by comparing results with those obtained from a region-growing algorithm. Discussion and projects are presented in Section 8.

\section{RELATED WORK}

The vessel segmentation methods can be classified according to the kind of strategy they use. The very first proposed methods were based on filtering [2]. Algorithms based on mathematical morphology (hysteresis thresholding in [3], grey-level erosions and dilations in [4], or grey-scale skeletonization in [5]) and region-growing [6] have also been proposed.

Among all the existing strategies, very few have been designed to take advantage of multimodal data. A method, proposed in [7], for cerebral vascular structures visualization uses both 3D MRA and 2D X-ray images. An algorithm has also been proposed in [1] where angiographic and non angiographic data are involved in an atlas-based region-growing algorithm. Nevertheless, the simultaneous use of images from different modalities, as the use of a priori anatomical knowledge, is quite unusual. The algorithm presented here, based on grey-level hit-or-miss transform [8] uses bimodal PC-MRA data and integrates a priori knowledge modeled by a vascular atlas.

\section{PC-MRA IMAGING}

PC-MRA is a non invasive 3D MR technique [9] enabling to generate two different images during a same acquisition. The first data, named magnitude image, contains anatomi- 

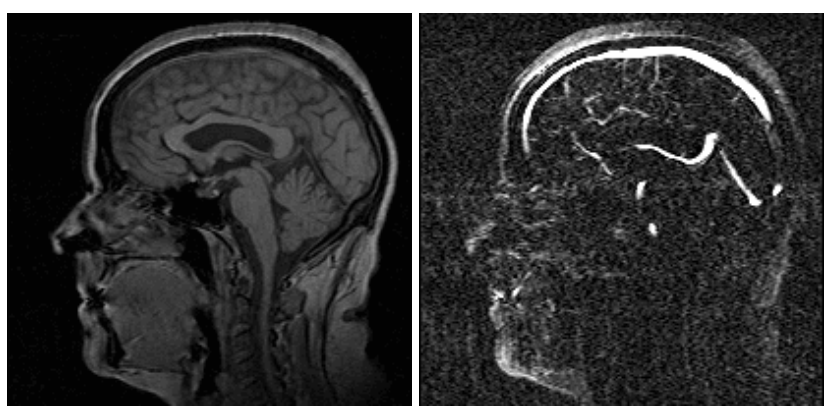

Fig. 1. PC-MRA sagittal slices. Left: magnitude image slice. Right: phase image slice. Since images are generated from the same acquisition, they are perfectly superimposed.

cal, but no vascular information, like a classical MRI. The second data, named phase image, is obtained by only conserving the signal of moving structures. Thus, the obtained image contains the flowing blood signal, plus noise and artefacts. Sagittal slices of such magnitude and phase images are illustrated in Fig. 1. The 30 MRA used in this paper have been performed on a 1 Tesla whole-body scanner (Gyroscan NT/INTERA 1.0 T from Philips, gradient slope 75 $\mathrm{T} / \mathrm{m} / \mathrm{s}$ ). The flow encoding sequence called T1FFE/PCA uses a TR of $10 \mathrm{~ms}$ and a TE of $6.4 \mathrm{~ms}$. The acquired images have dimensions varying between $256^{2} \times 150$ and $256^{2} \times 180$ voxels.

\section{ANATOMICAL KNOWLEDGE}

The vessel segmentation methods generally use very little a priori knowledge. Indeed, they are based on three main hypotheses: vessels correspond to the voxels of highest value in MRA; vessels have a tubular shape; vascular networks are organized in a tree structure. Nevertheless, brain vessels present other important properties. First, all the parts of the head do not contain the same quantity of vessels (this assumption takes into account the fact that MRA is unable to detect vessels having a diameter lower than $1 \mathrm{~mm}$ ). Second, the size and orientation of brain veins and arteries depend on their position. Then, in each brain or head area, homogeneous properties concerning vascular density and vessel size and orientation can be observed. This allows to create vascular atlases for modeling this knowledge and to use them to guide segmentation tools. In the following we propose to use such an atlas in order to guide grey-level hit-or-miss operators.

\section{GREY-LEVEL HIT-OR-MISS TRANSFORM}

The hit-or-miss transform is a classical tool for extraction of templates from binary images. It uses a couple of struc- turing elements $(A, B)$ where $A$ (resp. $B)$ has to fit the object (resp. the background). The binary hit-or-miss operation $(\otimes)$ can then be defined by:

$$
X \otimes(A, B)=\left\{p \in \varepsilon \mid A_{p} \subseteq X \wedge B_{p} \subseteq X^{c}\right\},
$$

where $X$ is a binary object of a given Euclidian or digital space $\varepsilon$ (in our case, $\varepsilon \subset \mathbb{N}^{3}$ ).

Any increasing operator can be extended to grey-level images by considering them as stacks of binary images. However, the hit-or-miss transform, as defined previously is not an increasing operation. Another definition ( $($ ) has then been proposed by Ronse in [8]:

$$
X \otimes(A, B)=\left\{p \in \varepsilon \mid A_{p} \subseteq X \subseteq B_{p}\right\},
$$

where $A$ and $B$ are structuring functions $(A \leq B)$. The greylevel hit-or-miss operation can then be defined by:

$$
F \oplus(A, B)=\bigvee\left\{i_{(p, t)} \mid A_{(p, t)} \leq F \leq B_{(p, t)}\right\},
$$

where $F$ is a grey-level function and $i_{(p, t)}$ is the impulse function defined by:

$$
i_{(p, t)}=\left\{\begin{array}{cl}
t & \text { if } x=p \\
-\infty & \text { if } x \neq p
\end{array} .\right.
$$

In [8], it has then been demonstrated that:

$$
[F \oplus(A, B)](p)=\left\{\begin{array}{cl}
(F \ominus A)(p) & \text { if }(F \ominus A)(p) \geq\left(F \oplus B^{*}\right)(p) \\
-\infty & \text { otherwise }
\end{array},\right.
$$

where $B^{*}$ is defined by $B^{*}(p)=-B(-p)$.

Choosing two structuring elements $A_{e}$ and $B_{e}$, and two grey-levels $a$ and $b$, with $a \geq b$, we can define the grey-level structuring elements $A$ and $B$ by:

$$
A(p)=\left\{\begin{array}{cc}
a & \text { if } p \in A_{e} \\
-\infty & \text { if } p \notin A_{e}
\end{array} \text {, and } B(p)=\left\{\begin{array}{cc}
b & \text { if } p \in B_{e} \\
+\infty & \text { if } p \notin B_{e}
\end{array}\right. \text {, }\right.
$$

and then obtain:

$$
F \ominus A=\left(F \ominus A_{e}\right)-a, \text { and } F \oplus B^{*}=\left(F \oplus \check{B}_{e}\right)-b,
$$

with $\check{B}=\{-b \mid b \in B\}$, and finally:

$$
[F \otimes(A, B)](p)=\left\{\begin{array}{cc|c|c|}
\left(F \ominus A_{e}\right)(p)-a & \text { if } \mid \begin{array}{l}
\left(F \ominus A_{e}\right)(p) \geq \\
\left(F \oplus \check{B}_{e}\right)+a-b \\
-\infty
\end{array} & \text { otherwise }
\end{array} .\right.
$$

Using this definition is equivalent to compare, at each point, the minimum intensity $a_{\min }$ of all points within $A_{e}$ and the maximum intensity $b_{\max }$ of all points within $B_{e}$. If $a_{\text {min }} \geq b_{\text {max }}+a-b$, then the point belongs to the transform.

\section{METHOD}

The proposed method is based on the use of the grey-level hit-or-miss transform. This mathematical morphology operator has already been used by Naegel et al. in [10] for the detection of the portal vein entry in liver CT data. A fixedsized operator was then described to fit this precise anatomical structure. In this paper, we propose to use the grey-level hit-or-miss transform to carry out a whole segmentation of the cerebral arterial and venous trees. 


\subsection{Shape of the structuring elements}

In order to fit the searched vascular structures, two kinds of structuring elements have been choosen. The element $A$, assumed to model the vessels, is a discrete sphere of radius $r_{A}$, while the element $B$, modeling the neighboring background, is a set of 6 points sampled from a circle of radius $r_{B}$, having the same center than $A$ and oriented according to angles $\left(\theta_{B}, \phi_{B}\right)$. A set of such structuring elements is illustrated in Fig. 2 (left picture). The choice of spheres instead of ellipsoids for vessel modeling is justified by their higher robustness in case of vessel tortuosity. Moreover, the use of points sampled from a circle instead of a whole discrete circle allows a better detection of the background even in case of vessel junctions or bifurcations, with a lower computation time.

\subsection{Use of the atlas}

The immediate approach consisting in applying every couple of structuring elements in each point of the studied MRA data $\left(D_{i}\right)$, to obtain the segmented image $\left(D_{s}\right)$ :

$D_{s}=\left\{p \in D_{i} \mid \exists\left(r_{A}, r_{B}, \theta_{B}, \phi_{B}, a, b\right),\left(D_{i} \ominus A\right)(p) \geq\left(D_{i} \oplus \check{B}\right)+a-b\right\}$,

leads to a prohibitive computational complexity. Since this strategy can easily require more than $10^{10}$ operator applications for one MRA data, it is necessary to reduce the algorithm complexity. This can be done by choosing, for each voxel, a subset of structuring elements sufficiently small to allow an efficient computation time and sufficiently large to find the vascular structures. This choice is done by taking into account anatomical knowledge modeled by a vascular atlas, the creation of which is fully detailed in [11]. This atlas can be defined by:

$$
\begin{array}{|cccc}
\mathcal{A}: & I & \rightarrow & {[0,1] \times \mathcal{P}(\mathbb{R}) \times \mathcal{P}([0, \pi[\times[0, \pi[)} \\
& \mathbf{x} & \mapsto & (d, T, O)
\end{array},
$$

where $\mathcal{P}(X)=\{Y \mid Y \subseteq X\}, d$ is the probability to find a vessel, $T$ is the set of possible vessel thicknesses and $O$ is the set of possible vessel orentations at the position $\mathbf{x} \in I$. In the following, $(d, T, O)$ will also be denoted by $\left(\mathcal{A}^{d}(\mathbf{x}), \mathcal{A}^{T}(\mathbf{x}), \mathcal{A}^{O}(\mathbf{x})\right)$. This atlas provides a statistical estimation of vessels parameters for each voxel of a reference image. It can then be used to determine subsets of structuring elements adapted to every voxel of the image to process.

\subsection{Algorithm}

The method takes as input a PC-MRA of the whole head, composed of both phase $(P)$ and magnitude $(M)$ images (see Fig. 1), the vascular atlas $(\mathcal{A})$, and the reference PC-MRA magnitude image $\left(M_{\mathcal{A}}\right)$ associated to the atlas.

The first step is the superimposition of $M_{\mathcal{A}}$ on $M$. This non rigid registration provides a $3 \mathrm{D}$ deformation field, used to obtain from $\mathcal{A}$ a new atlas $\mathcal{A}_{\text {def }}$ correctly fitting the processed MRA.

The second step is the choice of the set $S$ of voxels that will be processed by the hit-or-miss operators. This step is important for reducing the computation complexity since the vascular structures generally represent less than $3 \%$ of the image volume. Removing from $S$ all the voxels $\mathbf{x}$ such as $\mathcal{A}_{d e f}^{d}(\mathbf{x})=0$ enables to avoid processing the background or areas presenting no vessels such as the cerebellum or the skull.

The third step consists in determining, for all $\mathbf{x} \in S$, a subset of structuring elements and to apply them on the phase image $P$. For each $\mathbf{x} \in S$, this subset is created by choosing structuring elements $(A, B)$ such as $r_{A} \in \mathcal{A}_{d e f}^{T}(\mathbf{x})$, $\left(\theta_{B}, \phi_{B}\right) \in \mathcal{A}_{d e f}^{O}(\mathbf{x})$, and $\left.\left.r_{B} \in\right] r_{A}, 2 . r_{A}\right]$. Since the structuring elements are discrete objects, only discrete diameters and orientations (orientations according to discrete lines) are chosen. It has to be noticed that a fixed low value has been chosen for $a-b$, enabling to detect vascular structures presenting a low contrast with the background. All the operators $(A, B)$ are successively applied on $P$ at the position $\mathbf{x}$ until one (or no one) of them matches a structure. If a couple $(A, B)$ detects a vascular structure, the point $\mathbf{x}$ is added to the result image.

This method provides a skeleton image of the vascular tree. In order to obtain a volumic object, for each point $\mathbf{x}$ detected by a couple of structuring elements $(A, B)$, a dilation by $A$ is finally carried out. An example of such a segmented vascular tree is illustrated in Fig. 2 (right picture).

\section{RESULTS AND DISCUSSION}

The method has been implemented in $\mathrm{C}++$, and run on a computer using a $3 \mathrm{GHz}$ Pentium IV processor with $2 \mathrm{~GB}$ of memory. The average computation time is then 48 minutes to process one PC-MRA (38 minutes for the registration step and 10 minutes for the segmentation).

The 30 case database described in Section 3 has been used for validation. The results obtained with the proposed method have been quantitatively compared to those obtained with a previously described region-growing algorithm [1]. In average, $76 \%$ of vascular structures are correctly segmented by the proposed algorithm. The comparison also shows that the proposed method generates $23 \%$ of false negatives (vessels segmented by the region-growing method but missed by the proposed algorithm) but only $1 \%$ of false positives. This false positive ratio is quite satisfactory. Indeed, the method is essentially based on shape and size of structuring elements, but not on threshold values. Then it is not sensitive to non-tubular high-intensity artefacts which are generally the main cause of false positives. Moreover, by definition, the couple of structuring elements that enables to detect a vessel point immediately provides information 

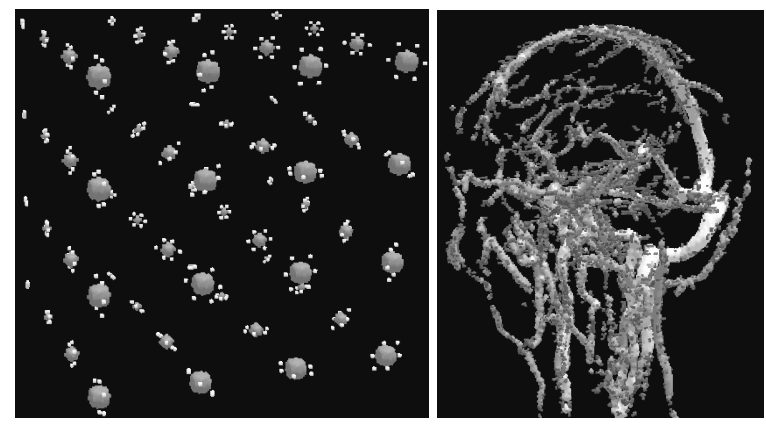

Fig. 2. Left: Part of the family of the structuring elements used to carry out the grey-level hit-or-miss segmentation. Right: Segmented vascular tree obtained with the proposed method. The grey-levels correspond to the radii of the structuring elements which permitted to segment the points.

on the vessel size and orientation, then avoiding a postprocessing quantification step. Nevertheless, this method still presents weaknesses concerning the false negative ratio. Indeed, it only uses spherical structuring elements to detect vessels. Then, the segmented vessels present tubular shape, even though their real shape is not tubular (many brain vessels have ellipsoidal or even triangular cross sections). Moreover, the smallest vessel are hardly segmented. For such structures, replacing the spherical structuring elements by line segments could provide better results.

\section{CONCLUSION}

This paper has presented a novel brain vessel segmentation method, based on grey-level hit-or-miss operators. The size and orientation of the operators are guided by anatomical knowledge modeled by a vascular atlas. This algorithm has been applied on a database of 30 PC-MRA, providing promising results. The method already provides a reliable vessel detection, presenting few false positive errors by comparison to a previously proposed region-growing algorithm. It also enables to directly obtain information on size and orientation of the segmented vessels. Its main weakness remains the unability to correctly reconstruct the smallest vessels or the vessels having a non circular cross-section. This problem is inherent to the current use of fixed-shaped spherical structuring elements. Then further work will now consist in adding the structuring elements shape as a supplementary parameter to be determined in each point of the image during the segmentation.

\section{REFERENCES}

[1] N. Passat, C. Ronse, J. Baruthio, J.-P. Armspach, C. Maillot, and C. Jahn, "Atlas-based method for segmentation of cerebral vascular trees from phasecontrast magnetic resonance angiography," in Image Processing 2004, 2004, vol. 5370 of SPIE Proc., pp. 420-431.

[2] Y.P. Du and D.L. Parker, "Vessel enhancement filtering in three-dimensional angiograms using long-range signal correlation," Journal of Magnetic Resonance Imaging, vol. 7, pp. 447-450, 1997.

[3] G. Gerig, T. Koller, G Székely, C. Brechbühler, and O. Kübler, "Symbolic description of 3-D structures applied to cerebral vessel tree obtained from MR angiography volume data," in Information Processing in Medical Imaging'93, 1993, vol. 687 of LNCS, pp. 94-111.

[4] H.E. Cline, D.R. Thedens, C.H. Meyer, D.G. Nishimura, T.K. Foo, and S. Ludke, "Combined connectivity and a grey-level morphological filter in magnetic resonance coronary angiography," Magnetic Resonance in Medicine, vol. 43, pp. 892-895, 2000.

[5] P.J. Yim, P.L. Choyke, and R.M. Summers, "Grayscale skeletonization of small vessels in magnetic resonance angiography," IEEE Transactions on Medical Imaging, vol. 19, pp. 568-576, 2000.

[6] C. Zahlten, H. Jürgens, C.J.G. Evertsz, R. Leppek, H.O. Peitgen, and K.J. Klose, "Portal vein reconstruction based on topology," European Journal of Radiology, vol. 19, pp. 96-100, 1995.

[7] A.R. Sanderson, D.L. Parker, and T.C. Henderson, "Simultaneous segmentation of MR and Xray angiograms for visualization of cerebral vascular anatomy," in Volume Image Processing'93, 1993.

[8] C. Ronse, "A lattice-theoretical morphological view on template extraction in images," Journal of Visual Communication $\mathcal{E}$ Image Representation, vol. 7, pp. 273-295, 1996.

[9] C.L. Dumoulin, S.P. Souza, M.F. Walker, and W. Wagle, "Three-dimensional phase contrast angiography," Magnetic Resonance in Medicine, vol. 9, pp. 139-149, 1989.

[10] B. Naegel, C. Ronse, and L. Soler, "Using grey-level hit-or-miss transform for segmenting the portal network of the liver," To appear in 7th International Symposium on Mathematical Morphology - ISMM'05.

[11] N. Passat, C. Ronse, J. Baruthio, J.-P. Armspach, and C. Maillot, "Cerebral vascular atlas generation for anatomical knowledge modeling and segmentation purpose," Submitted. 